\title{
PREVALENCIA DE FACTORES DE RIESGO CEREBRO- CARDIOVASCULARES EN ESTUDIANTES DE SALUD, ARMENIA-QUINDÍO 2014
}

\author{
PREVALENCE OF BRAIN-RELATED CARDIOVASCULAR RISK \\ FACTORS IN HEALTH STUDENTS, ARMENIA-QUINDÍO 2014
}

\begin{abstract}
1 Enfermera. Especialista en Promoción de la Salud. Magister en Salud Pública. Docente del programa de enfermería, facultad de ciencias de la salud de la Universidad del Quindío.

2 Enfermera. Especialista en Gerencia de la Calidad y Auditoria en Salud. Magister en Salud Pública. Docente del programa de enfermería, facultad de ciencias de la salud de la Universidad del Quindío.jheimyjacke@gmail.com.

Recibido: 2 Diciembre de 2015

Aceptado: 22 Diciembre de 2015

*Correspondencia del autor: Diana Patricia Londoño Buriticá. Carrera 19 Nro.

10N43 Badajoz. Apartamento C 606. E-mail: dplondono@uniquindio.edu.co
\end{abstract}

\section{RESUMEN}

Las enfermedades cerebro-cardiovasculares representan la principal causa de muerte en el mundo en personas mayores de 45 años; en los últimos tiempos estos padecimientos se reconocen en población joven. Estas son enfermedades crónicas no transmisibles, que se relacionan principalmente con factores de riesgo modificables propios de los estilos de vida, aunque existe un componente hereditario que puede estar asociado a la aparición de la enfermedad.

El presente estudio cuantitativo, descriptivo, tuvo como objetivo medir la prevalencia de los factores de riesgo de enfermedades cerebro-cardiovasculares que tradicionalmente se han contemplado en la literatura. Se midieron edad, sexo, etnia y antecedentes familiares como factores de riesgo no modificables, y consumo elevado de alcohol, consumo de cigarrillo, inactividad física, Índice de Masa Corporal elevado y tensión arterial alta por toma casual, como factores de riesgo modificables.

La inactividad física $(87,8 \%$ ) y el consumo alto de alcohol $(46,3 \%)$ se identificaron como los factores de riesgo modificables con mayor prevalencia en el grupo de jóvenes de 15 a 25 años. Se identificaron estudiantes con antecedentes familiares de enfermedades cerebro-cardiovasculares y personales de hipertensión arterial y de diabetes mellitus. Como hallazgo emergente del estudio, se identificó un 6,5\% de la población estudiantil con bajo peso $(\mathrm{IMC}<18)$.

Se contrastaron los datos con algunos de investigaciones similares nacionales e internaciones, encontrándose factores de riesgo modificables con prevalencias más altas, que las reportadas en los mismos.

Palabras claves: Prevalencia, Factores de riesgo, Cerebrovascular, Cardiovascular, Estudiantes universitarios. 


\begin{abstract}
Cerebrovascular and cardiovascular diseases are the leading cause of death in people over forty-five years in the world. Recently, these conditions are also found in young people. These are chronic and non-communicable illnesses that are mainly related with modifiable risk factors inherent to lifestyles. However, there is a hereditary component that can be associated with the disease onset.
\end{abstract}

This quantitative and descriptive study aimed to measure the prevalence of cerebrovascular and cardiovascular diseases risk factors that have been traditionally considered in the literature. Age, gender, ethnicity and family history were measured as non-modifiable risk factors. On the other hand, high alcohol consumption, cigarette smoking, physical inactivity, high body mass index and casual high blood pressure measurement, were assessed as modifiable risk factors.

The most prevalent modifiable risk factors that were identified in the group of young people between fifteen and twenty five years old were physical inactivity $(87,8 \%)$ and high alcohol consumption (46,3\%). Students with family history of cerebrovascular and cardiovascular diseases, with personal history of high blood pressure and diabetes mellitus were also recognized. As an emerging finding of the study, it was identified that $6,5 \%$ of the student population was underweight $(\mathrm{BMI}<18)$

Data were compared with some similar national and international researches, finding modifiable risk factors with higher prevalence than the ones reported in our investigation.

Keywords: Prevalence, risk, cerebrovascular, cardiovascular, university students.

\section{INTRODUCCIÓN}

Las enfermedades cerebro-cardiovasculares ECCV (agrupación de enfermedades cerebrales y del corazón, que se producen a partir del daño de los vasos sanguíneos, especialmente las arterias) son enfermedades crónicas, no transmisibles de gran relevancia para la salud pública por representar una gran carga de morbilidad y por considerarse como la principal causa de muerte en el mundo.

Se estima que aproximadamente 1 de cada 3 personas, es decir, alrededor de 17.5 millones, fallecen anualmente por cualquier afección de este grupo, entre las que más se presentan son las enfermedades isquémicas del corazón y las enfermedades hipertensivas. Para éstas últimas el American Heart Association (2012) estima "en 7,1 millones el número mundial de muertes, cifra que representa aproximadamente el $13 \%$ de la mortalidad total ${ }^{1}$.

Las ECCV se presentan tanto en países desarrollados, como en aquellos poco industrializados. La Federación Internacional de Diabetes (2014) comenta que

1. American Heart Association. [Internet] 2012 [Consultado marzo 2014]; Disponible en: URL: http://www.americanheart.org/presenter. jhtml?identifier $=3001008$ "las muertes por estas causas afectan por igual a ambos sexos y más del $80 \%$ se producen en países de ingresos bajos y medios ${ }^{2}$ ". La transición demográfica y epidemiológica, el aumento de la esperanza de vida, la disminución de los nacimientos y un estilo de vida cada más sedentario, representan las causas directas de dichos eventos. Para el año 2011 la tasa de mortalidad por enfermedades cerebrocardiovasculares ECCV en Colombia, fue de 392,5/100.000 habitantes y para el año 2012, fue de 532,39/100.000 habitantes, según el Ministerio de Salud y de la Protección Social (2011-2012).

Las enfermedades cerebro-cardiovasculares (ECCV) no son la consecuencia de un solo factor de riesgo aislado, sino que representan el resultado final de un proceso que comienza tempranamente en la vida e involucra muchos otros factores: conductuales, ambientales, socioeconómicos y genéticos. Parece lógico entonces, que para prevenir estas entidades, sea necesario controlar varios factores de riego y sus determinantes, simultáneamente.

Se consideran factores de riesgo no modificables

2. International Diabetes Federation. [Internet] [Consultado julio 2014]; Disponible en: URL: http://www.idf.org 
aquellas circunstancias propias de cada individuo que lo hacen vulnerable a padecer alguna de estas enfermedades y que por sus características, no pueden ser controladas ni disminuidas, entre ellos se encuentran la edad, el sexo, la etnia y los antecedentes familiares de ECCV. Si a éstas se suman factores de riesgo modificables, se potencia la posibilidad de padecer la enfermedad.

Los factores de riesgo modificables, hacen referencia generalmente a comportamientos de los individuos o de grupos poblacionales, conocidos como hábitos poco saludables y que se relacionan con el aumento o la predisposición al padecimiento de dichas enfermedades, que pueden ser prevenibles e intervenibles. Dichos factores son: la hipertensión arterial, la diabetes mellitus, el consumo de cigarrillo, el alto consumo de licor, la inactividad física, el inadecuado manejo del estrés, el sobrepeso u obesidad y las dislipidemias.

Según el Texas Heart Institute (2015) los factores de riesgo principales son: la hipertensión arterial, el aumento de los niveles de colesterol en sangre, la diabetes mellitus, la obesidad y/o sobrepeso, el consumo de cigarrillo, el sedentarismo, el sexo, la edad y la herencia. Los factores de riesgo contribuyentes son: el inadecuado afrontamiento del estrés y el consumo de licor ${ }^{3}$.

Fue de esta manera, como el presente estudio tuvo como objetivo, identificar la prevalencia de los factores de riesgo ECCV en los jóvenes de 15 a 25 años, estudiantes de enfermería y de medicina de la Facultad de salud de una Universidad pública del Quindío, año 2014. A partir de un estudio cuantitativo, observacional, descriptivo de corte transversal.

\section{MATERIALES Y MÉTODOS}

Estudio cuantitativo, observacional, descriptivo de corte transversal, que fue evaluado y avalado por los comités técnico y científico y de bioética de la Universidad donde se llevo llevó a cabo.

La población participante (tipo censo) estuvo conformada por 231 estudiantes activos y matriculados de los programas de enfermería y medicina de la Facultad de Salud de una universidad del Quindío entre los años 2013 y 2014 . Hombres y mujeres entre 15 y 25 años, incluidos aquellos con diagnóstico previo de hipertensión arterial y diabetes mellitus; no se incluye- ron mujeres embarazadas.

Se realizó prueba piloto con estudiantes de la Facultad de Ciencias Sociales de la misma Universidad.

La recolección de la información se realizó en un plazo de 10 semanas, previo consentimiento de los directores de los programas de enfermería y medicina. Los estudiantes fueron abordados dentro de la misma facultad, previa revisión de la base de datos suministrada por la oficina de admisiones y registro.

Se aplicó cuestionario compuesto por 29 preguntas, además de la medición del peso, la talla y la tensión arterial, según protocolos elaborados para dichos fines, previa firma del consentimiento informado.

Se construyó base de datos. Se realizó análisis univariado identificando las prevalencias de los factores de riesgo modificables y no modificables; y análisis bivariado a través de la construcción de tablas de $2 \times 2$, OR aplicación de pruebas Chi2 $\left(\mathrm{X}^{2}\right)$ o test exacto de Fisher con sus valores de $\mathrm{P}$.

\section{RESULTADOS}

La población sujeto de estudio estuvo conformada por 301 estudiantes que cumplían criterios de inclusión, de los cuales se logró la participación de 231 con un $76,6 \%$.

Tabla 1: Variables sociales y demográficas del grupo de estudiantes participantes en el estudio (Denominador: 231)

\begin{tabular}{ccccc}
\hline \multirow{4}{*}{ Programa } & $\mathbf{2 0 - 2 5 \text { años }}$ & $\mathbf{1 1 9}$ & $\mathbf{5 1 , 6}$ & $\mathbf{( 4 4 , 8}$ a 58,0) \\
\cline { 2 - 5 } & Enfermería & 151 & 65,4 & $(58,8$ a 71,4) \\
& Medicina & 80 & 34,6 & $(28,5$ a 41,2) \\
\multirow{4}{*}{ Semestre } & Primer Ciclo & 173 & 75 & $(68,7$ a 80,2) \\
& Segundo Ciclo & 58 & 25 & $(19,7$ a 31,3) \\
& Negro & 6 & 2,6 & $(1,0$ a 5,8) \\
& Blanco & 27 & 11,6 & $(7,9$ a 16,7) \\
& Mestizo & 196 & 84,8 & $(79,4$ a 89,0) \\
& Indígena & 2 & 0,8 & $(0,1$ a 3,4) \\
\hline
\end{tabular}

Respecto al grupo de encuestados, se evidenció que éste estuvo representado en su mayoría, por mujeres $(n=169)$, con un 73,1\% (IC al 95\%=66,8\% a 78,6\%). Con respecto a los grupos de edad, estos se encuentran en porcentajes similares: grupo de 15 a 19 años con un $48,1 \%$ y grupo de 20 a 25 años con un $51,6 \%$. 
Un $84,8 \%$ (IC al 95\%=79,4\% a 89,0\%) de los participantes se representó en el grupo étnico mestizo. Sólo un 2,6\% (IC al 95\%=1,0\% a 5,8\%) de los estudiantes se representó como afrodescendiente (Negro).

El grupo de estudiantes del programa de enfermería, dentro de la muestra, tuvo una representación de un $65,3 \%$ (IC al $95 \%=50,8 \%$ a $71,4 \%$ ). Los estudiantes cursaban en su mayoría el primer ciclo: de primero a quinto semestre con un $75 \%$ (IC al $95 \%=68,7 \%$ a $80,2 \%$ ). Un $25 \%$ (IC al 95\%= 19,7\% a $31,3 \%$ ) restante correspondieron al segundo ciclo: de sexto semestre en adelante.

A continuación, se presentan los hallazgos relacionados con las prevalencias de factores de riesgo tradicionales de enfermedad cerebro-cardiovascular y los criterios de definición de los mismos (tabla 2).

Tabla 2: Prevalencia de factores de riesgo modificables y no modificables, de enfermedad cerebro-cardiovascular en estudiantes de la facultad de ciencias de la salud, Universidad Quindío 2014.

\begin{tabular}{|c|c|c|c|c|c|}
\hline Variable & Definición Estudio & $\mathbf{n}$ & Frecuencia & Prevalencia & IC $95 \%$ \\
\hline Antecedentes Familiares Presentes & * & 231 & 68 & $29,4 \%$ & $\begin{array}{l}23,7 \% \\
\mathrm{a} \\
35,8 \%\end{array}$ \\
\hline Diagnóstico Médico de HTA & $\begin{array}{l}\text { Auto-reportada por DX } \\
\text { médico y/o toma medica- } \\
\text { mentos }\end{array}$ & 231 & 4 & $1,7 \%$ & $\begin{array}{l}0,5 \% \\
\mathrm{a} \\
4,6 \%\end{array}$ \\
\hline Diagnóstico Médico de DM & $\begin{array}{l}\text { Auto-reportada por DX } \\
\text { médico y/o toma medica- } \\
\text { mentos }\end{array}$ & 231 & 1 & $0,4 \%$ & $\begin{array}{l}0,0 \% \\
\quad \mathrm{a} \\
2,7 \%\end{array}$ \\
\hline Inactividad física & $* *$ & 231 & 203 & $87,8 \%$ & $\begin{array}{l}82,8 \% \\
\quad a \\
91,6 \%\end{array}$ \\
\hline Alto Consumo de Alcohol & $\begin{array}{l}\text { Consumo de licor al me- } \\
\text { nos } 1 \text { vez en el último } \\
\text { mes. En la última oportu- } \\
\text { nidad Hombres más de } 28 \\
\text { gramos y mujeres más de } \\
\qquad 14 \text { gramos. }\end{array}$ & 231 & 107 & $46,3 \%$ & $\begin{array}{l}39,7 \% \\
\mathrm{a} \\
52,9 \%\end{array}$ \\
\hline Ebriedad Alto Consumo Alcohol & $\begin{array}{c}\text { Consumo de alcohol hasta } \\
\text { la ebriedad }\end{array}$ & 107 & 47 & $43,9 \%$ & $\begin{array}{l}34,4 \% \\
\mathrm{a} \\
53,8 \%\end{array}$ \\
\hline Fumador & $\begin{array}{l}\text { Haber fumado más de } 100 \\
\text { cigarrillos en la vida y el } \\
\text { último, fumado en los úl- } \\
\text { timos } 6 \text { meses }\end{array}$ & 231 & 3,9 & $3,9 \%$ & $\begin{array}{l}1,9 \% \\
\quad \mathrm{a} \\
7,5 \%\end{array}$ \\
\hline IMC Alto & $\begin{array}{l}\text { IMC Alto: sobrepeso y } \\
\text { obesidad, personas con } \\
\text { IMC mayor a } 24,9 \text {. }\end{array}$ & 231 & 36 & $15,5 \%$ & $\begin{array}{c}11,2 \% \\
a \\
21,0 \%\end{array}$ \\
\hline TA Alta & Por toma casual & 231 & 17 & $7,3 \%$ & $\begin{array}{c}4,4 \% \\
\mathrm{a} \\
11,7 \%\end{array}$ \\
\hline
\end{tabular}

* Familiares en primer grado de consanguinidad (Padres o Hermanos) con antecedente de enfermedad cerebro-cardiovascular, hombre menor de 55 años y/o mujer menor de 65 años.

** Inactividad física: persona que no haya realizado actividad física en la última semana, o aquella persona que haya realizado actividad 1 o 2 días en la última semana, o que realizando actividad física al menos 3 veces en la última semana pero la duración haya sido inferior a 30 minutos por día (Menos de 90 minutos por semana) con una intensidad moderada o enérgica. La intensidad de la actividad física se consideró, a partir de los siguientes criterios: i) Leve, como caminar. ii) Moderadas, aparece sudor en los primeros 10 minutos de la actividad. iii) Enérgicas, además de sudor, falta el aliento. 
Factores de riesgo no modificables:

a. Antecedentes familiares en primer grado de consanguinidad de enfermedad cerebro-cardiovascular temprana: Los antecedentes familiares de enfermedad cerebro-cardiovascular en primer grado de consanguinidad, representaron la principal prevalencia de factores de riesgo tradicionales no modificables en la población de estudiantes a riesgo, con un $29,4 \%$ (IC al $95 \%=23,7 \%$ a $35,8 \%$ ).

Los antecedentes familiares de ECCV se encuentran en el $40,3 \%$ de los hombres (IC al $95 \%=28,3 \%$ a $53,5 \%$ ) y en mujeres del $25,4 \%$ (IC al $95 \%=19,2$ a $32,8 \%$ ). Existen diferencias significativas en esta variable según sexo $\left(\mathrm{X}^{2} 1\right.$ g.l. 4,12 ; valor $\left.\mathrm{p}=0,04\right), \mathrm{RP}=$ $1,58$ (IC al $95 \%=1,6$ a 2,36$)$.

Llama la atención encontrar prevalencias tan altas en el grupo de estudiantes.

\section{Según el Texas Heart Institute}

"las enfermedades del corazón suelen ser hereditarias. Si padres o hermanos padecieron de un problema cardíaco o circulatorio antes de los 55 años de edad, la persona tiene un mayor riesgo cardiovascular que alguien que no tiene esos antecedentes familiares. Los factores de riesgo tales como la hipertensión, también pueden transmitirse de una generación a la siguiente ${ }^{4}$ ".

b. Diagnóstico médico de hipertensión arterial: La prevalencia de hipertensión arterial diagnosticada por medico con o sin tratamiento farmacológico, en la población a riesgo fue de $1,7 \%$ (IC al $95 \%=0,5 \%$ a $4,6 \%)$.

La hipertensión arterial se encuentra en el 4,8\% de los hombres (IC al $95 \%=1,2 \%$ a $14,3 \%$ ) y en mujeres la prevalencia fue de $0,5 \%$ (IC al $95 \%=0,0 \%$ a 3,7 ). No se hallaron diferencias significativas según sexo.

c. Diagnóstico médico de diabetes mellitus: En los estudiantes a riesgo diagnosticados con diabetes mellitus se presentó una prevalencia de $0,4 \%$ (IC al $95 \%=0,0 \%$ a $2,7 \%$ ) representada en una persona.

El caso estuvo representado por una mujer mestiza de

4. Texas Heart Institute. [Internet] [Consultado marzo 2015]; Disponible en: URL: http://www.texasheartinstitute.org/HIC/Topics_Esp/HSmart/ riskspan.cfm
22 años de edad, estudiante de enfermería del segundo ciclo. Diagnosticada en el año 2.003 y desde ese momento se encuentra en tratamiento farmacológico. No es hipertensa y no tiene antecedentes familiares de ECVV.

Los hallazgos sobre los factores de riesgo no modificables, brindan un panorama general de la situación actual de los mismos en la población participante. Si bien este tipo de factores de riesgo no pueden ser intervenidos, la presencia de antecedentes familiares potencializada por factores de riesgo modificables, marcan una tendencia favorecedora del desarrollo de enfermedad cerebro-cardiovascular.

Según el Texas Heart Institute (2015) el riesgo es acumulativo a lo largo de la vida. A más factores de riesgo y mayor edad, mayor probabilidad de que suceda el evento.

\section{Factores de riesgo modificables:}

a. Inactividad física habitual: La inactividad física habitual fue el factor de riesgo modificable tradicional con la prevalencia más alta con un $87,8 \%$ (IC al $95 \%=$ $82,8 \%$ a $91,6 \%$ ) representado en 203 personas.

Estos hallazgos sobre la inactividad física son muy importantes si se tiene en cuenta que la "inactividad física es el cuarto factor de riesgo de mortalidad en el mundo, según la OMS (2010) ${ }^{5 \%}$. La práctica de actividad física regular se relaciona con mejores niveles de salud de las personas, mejora el pronóstico y la evolución de los hipertensos y de los individuos que han tenido episodios cardiovasculares. Ayuda además a mantener el control del peso corporal y mejora la salud mental en personas sanas y/o enfermas.

b. Consumo alto de alcohol: El alto consumo de alcohol es el segundo factor de riesgo tradicional modificable más prevalente en el grupo de estudiantes a riesgo, con un 46,3\% (IC al 95\%=39,7\% y 52,9\%).

El consumo de alcohol alto no presenta diferencias significativas entre el grupo de hombres y mujeres, tampoco por grupos de edad o programa académico.

5. World Health Organization. Global status report on noncommunicable diseases 2010. WHO Library Cataloguing-in-Publication Data, 2011. ISBN 9789240686458 
La importancia de este factor de riesgo tradicional modificable, radica además en el hallazgo del componente de ebriedad. Un $44 \%$ de todos los estudiantes que consumieron licor en el mes anterior al diligenciamiento de la encuesta, manifestaron hacerlo hasta la ebriedad, lo que genera mayores implicaciones para la salud física y mental del individuo.

c. Índice de masa corporal IMC: La prevalencia de la variable índice de masa corporal alto fue de un $15,5 \%$ (IC al $95 \%=11,2 \%$ a $21,0 \%$ ), incluyendo aquellos datos que revelan sobrepeso y obesidad.

El índice de masa corporal IMC, es una medida de obesidad que relaciona el peso y la talla de los individuos. Según la Organización Mundial de la Salud OMS, un IMC mayor o igual a 25, se clasifica como sobrepeso y un IMC mayor o igual a 30 , se clasifica como obesidad.

Los datos de IMC alto, se presentaron de la siguiente forma: sobrepeso (IMC $>24,9$ y $<30$ ) 11,6\% (IC 95\%= $7,9 \%$ a $16,7 \%$ ) y obesidad grado I (IMC $>29,9$ y $<35$ ) $3,9 \%$ (IC 95\%=1,9\% a 7,5\%). No existen diferencias significativas para esta variable, según el sexo.

Llama la atención un hallazgo dentro del presente estudio, se relaciona con la prevalencia del IMC $<18.0$ en un $6,5 \%$ de la población de estudio (IC al $95 \%=3,8 \%$ a $10,7 \%$ ). Sin embrago estos valores no pueden ser contrastados, por carecer de datos en otros estudios. d. Consumo de cigarrillo: La prevalencia general de consumo de cigarrillo fue 3,9\% (IC al $95 \%=1,9 \%$ a $7,5 \%$ ). En hombres fue 4,8\% (IC al $95 \%=1,2 \%$ a $14,3 \%$ ) y en mujeres $3,5 \%$ (IC al $95 \%=1,4 \%$ a $7,9 \%$ ).

No existe diferencia significativa para esta variable, según sexo y programa académico.

e. Tensión arterial alta: En el caso de las cifras tensionales elevadas por toma casual, se encontró una prevalencia de un 7,3\% (IC al 95\%=4,4\% a $11,7 \%)$.

Los datos de tensión arterial se encuentran en el $16,1 \%$ de los hombres (IC al 95\%=8,4\% a $28,1 \%$ ) y en mujeres del $4,1 \%$ (IC al $95 \%=1,8$ a $8,6 \%$ ). Existen diferencias significativas en esta variable según sexo $\left(\mathrm{X}^{2} 1\right.$ g.l. 7,88; valor $\left.\mathrm{p}=0,00\right), \mathrm{RP}=$ 3,49 (IC al $95 \%=1,55$ a 9,78 ).

Aunque los hallazgos sobre la medición de la tensión arterial no son datos equiparables con diagnóstico de hipertensión arterial, es preocupante encontrar cifras del 7,3\% y más aún, cuando es más alta en el grupo de hombres con un 16,1\%. Adicionalmente, aquellos estudiantes que efectivamente son hipertensos y aun no lo saben, no han iniciado tratamiento farmacológico, ni han asumido cambios en su estilo de vida, que los ex-

En resumen, del grupo de estudiantes encuestados se puede observar:

Tabla. 3: Prevalencia de factores de riesgo modificables y no modificables, de enfermedad cerebro-cardiovascular en estudiantes de la facultad de ciencias de la salud, Universidad Quindío 2014.

\begin{tabular}{cccc}
\hline F. R. No Modificables & $\mathbf{n}$ & Prevalencia & IC 95\% \\
\hline Estudiantes sin F. Riesgo & 123 & $53,2 \%$ & 46,6 a 59,7 \\
Estudiantes con 1 F. Riesgo & 81 & $35,0 \%$ & 29,0 a 41,6 \\
Estudiantes con 2 F. Riesgo & 26 & $11,2 \%$ & 7,6 a 16,2 \\
Estudiantes con 3 F. Riesgo & 1 & $0,4 \%$ & 0,0 a 2,7 \\
F. R. Modificables & $\mathbf{n}$ & Prevalencia & IC 95\% \\
Estudiantes con 1 F. Riesgo & 116 & $50,2 \%$ & 43,6 a 56,8 \\
Estudiantes con 2 F. Riesgo & 76 & $32,9 \%$ & 26,9 a 39,4 \\
Estudiantes con 3 o más F. Riesgo & 39 & $16,8 \%$ & 12,4 a 22,4 \\
& & & 1,6 a 6,9 \\
\hline Estudiantes libres de F. Riesgo & 8 & $3,4 \%$ &
\end{tabular}


ponen a sufrir complicaciones mayores; más aun, este tipo de padecimientos son más frecuentes en personas adultas de mayor edad y se relacionan frecuentemente con patrones de conductas que pueden ser identificadas y contrarrestadas a tiempo.

Así, se evidencia que la prevalencia de los factores de riesgo de ECCV es muy alta para la población de estudio y que pese a los conocimientos en salud, los factores de riesgo modificables son los más frecuentes.

\section{Análisis bivariado:}

Para el análisis bivariado se definieron las variables que se suponían como factores de riesgo y aquellas de resultado.

En este caso las variables consideradas como factores de riesgo, fueron: edad, sexo, etnia, programa académico, semestre y antecedentes familiares.

Las variables consideradas como resultado, fueron: hipertensión arterial, diabetes mellitus, consumo de licor, consumo de cigarrillo, inactividad física, tensión arterial e índice de masa corporal. Con respecto a la variable ebriedad, esta sólo fue considerada para el grupo de personas con consumo de licor.

Con el fin de establecer la existencia de asociación entre las variables consideradas factores de riesgo y las variables consideradas de respuesta, se construyeron tablas de $2 \times 2$ y se aplicaron pruebas de asociación: OR, y pruebas de significancia estadística: Chi2 $\left(\mathrm{X}^{2}\right)$ y test exacto de Fisher.

Se calcularon O.R. (Odds Ratio) y su respectivo intervalo de confianza para cada una de las 35 tablas construidas, buscando asociación entre las variables analizadas (magnitud de la asociación). Las pruebas de Chi2 $\left(\mathrm{X}^{2}\right)$ y test exacto de Fisher se usaron para determinar si se encontraba asociación estadísticamente significativa.

Las pruebas de Chi2 $\left(\mathrm{X}^{2}\right)$ muestran el valor calculado para el mismo y el valor $\mathrm{P}$ correspondien- te con un grado de libertad. La regla de decisión para rechazar la hipótesis nula (Ho: No hay deferencia significativa entre los grupos observados) fue un valor $P$ igual o inferior a 0,05 .

El test exacto de Fisher se calculó para aquellas tablas de $2 \times 2$ en donde los valores esperados en alguna de las casillas fueron de 5 o menos. Esta prueba muestra los valores del $\mathrm{P}$ calculado.

Para comparar los resultados obtenidos en la investigación con los datos obtenidos en otros estudios, se calcularon Razones de Prevalencias R.P con su respectivo intervalo de confianza al $95 \%$.

Finalmente, los datos de este análisis muestran que ninguna de las relaciones entre las variables "factores de riesgo" y "resultado" es estadísticamente significativa.

\section{DISCUSIÓN}

Los hallazgos obtenidos en la presente investigación, son alarmantes. Al realizar comparaciones con otros estudios, se evidencian prevalencias más altas en factores de riesgo modificables, así:

Los niveles de inactividad física en la población universitaria estudiada $(87,8 \%)$, podrían explicarse por la dinámica del pregrado. Las carreras de ciencias de la salud exigen ocupación del mayor tiempo posible, por parte de los estudiantes, así, las ocupaciones, las presiones y las exigencias de las carreras, limitan el tiempo libre de los estudiantes, momentos en los cuales se podrían disponer a realizar actividad física de forma regular.

Según los indicadores básicos para Colombia en el año 2011 se reportaron datos de inactividad física para población de 18 a 64 años con una prevalencia del $54,8 \%$ (hombres $63,5 \%$ y mujeres 46,1\%). Para América Latina los datos según OMS (2010) son del 50\% para mujeres y $40 \%$ para hombres, en la población general.

Al comparar los resultados obtenidos con resul- 
tados de estudios similares, se hallan diferencias significativas (X2 1 g.1. 54,8; valor $\mathrm{p}=0,00$ ), entre las prevalencias de inactividad física para hombres y mujeres de 14 a 19 años en el estudio de Beck y colaboradores, Brasil (2011) (61,2\%) y las prevalencias del presente estudio $(87,8 \%)$ siendo ésta últimas más altas, $\mathrm{RP}=0,70$ (IC al $95 \%=0,64$ a 0,75$)$.

De igual forma la $\mathrm{RP}=0,88$ (IC al $95 \%=0,81$ a $0,94)$ hallada en la comparación de los resultados de inactividad física del presente estudio $(87,8 \%)$ con los datos para la ciudad de Cartagena, estudio de Guerrero y colaboradores (2009) $(76,9 \%)$, muestran diferencias significativas $\left(\mathrm{X}^{2} \quad 1\right.$ g.l. 10,10 ; valor $p=0,00$ ) encontrando prevalencias más altas para los estudiantes universitarios encuestados en la ciudad de Armenia.

En el presente estudio, los hombres y mujeres encuestados tienen prevalencias similares de inactividad física $87 \%$ y $88 \%$ respectivamente, diferente a lo que muestran estudios para Chile (Chiang, 1999), prevalencia de inactividad física en hombres del $47,8 \%$ y para mujeres del $73,7 \%$. Reportes de Brasil (Cazuza de Farias, 2010) muestran prevalencias de inactividad física en hombres del 50,7\% y para mujeres del 66,8\%. En las tendencias y los reportes a nivel mundial y de Latinoamérica, los hombres son más activos que las mujeres (OMS 2010).

Con respecto al consumo de alcohol, los presentes hallazgos (prevalencia 46,3\%) podrían darse por las facilidades que tienen los estudiantes de la universidad para adquirir las bebidas alcohólicas.

Además los estudiantes universitarios pueden tener un poder adquisitivo mayor que otros jóvenes $\mathrm{y}$ al vivir solos o con grupos de amigos, puede ejercerse menor control por parte de sus familias.

Según los indicadores de salud del año 2011, para Colombia, el consumo de alcohol (último mes, en el momento de la encuesta) fue del $46,06 \%$ para hombres y del $24,83 \%$ en mujeres de 12 a 65 años.
Un estudio de Cartagena (2009) reportó en 86,9\% la prevalencia de consumo de alcohol, para hombres y mujeres. Al comparar los datos del presente estudio con los datos reportados para Cartagena, se halla una $\mathrm{RP}=1,87$ (IC al $95 \%=1,62$ a 2,17 ) con diferencias significativas $\left(\mathrm{X}^{2} 1\right.$ g.l. 107,65; valor $\mathrm{p}=0,00)$. Llama la atención que para dicha ciudad la prevalencia de consumo de alcohol, sea más alta que la reportada en el presente estudio.

Dado el enfoque de factores de riesgo tradicionales para enfermedad cerebro-cardiovascular, el Texas Heart Institute muestra como el alto consumo de alcohol se convierte en un riesgo para el padecimiento de la misma. El consumo moderado de alcohol (menos de 14 gramos/día en mujeres o menos de 28 gramos/día en hombres) se ha descrito como un factor cardioprotector, especialmente el consumir vino tinto a diario, según la American Heart Association (2011) y la OMS. Para efectos del presente estudio, se tuvo en cuenta como factor de riesgo aquel consumo señalado como alto dentro del último mes.

Las prevalencias de consumo de cigarrillo reportadas por el presente estudio no son inferiores a las reportadas en estudios nacionales e internacionales (Cartagena, Brasil). Al comparar los datos del con los reportados para Cartagena (2009) $(12,7 \%)$, se halla una $\mathrm{RP}=3,25$ (IC al $95 \%=1,62$ a 6,52$)$ con diferencias significativas $\left(X^{2} 1\right.$ g.l. $11,75$; valor $\mathrm{p}=0,00)$. Llama la atención que en esta ciudad (Cartagena) la prevalencia de consumo de cigarrillo, sea más alta que la reportada en el presente estudio.

Los datos de las prevalencias de hipertensión arterial reportada $(1,7 \%)$ e Índice de Masa Corporal IMC alto $(15,5 \%)$ no pudieron ser contrastados con otros estudios, por carecer de información para este grupo específico de edad, en relación a estudios de Chile año 1999, Cartagena año 2009 y Brasil año 2010.

\section{Conclusiones}

Las enfermedades cerebro-cardiovasculares ECCV son la principal causa de muerte en el 
mundo entero y generan una gran carga de morbilidad. En su informe del año 2010 sobre las enfermedades crónicas no transmisibles la OMS ha hablado de una epidemia mundial, para la cual reporta cuatro principales factores de riesgo: i) Consumo de tabaco. ii) Práctica insuficiente de actividad física. iii) Consumo inmoderado de alcohol (perjudicial). iv) Alimentación poco sana (aumento en el consumo de sal, azucares, grasas saturadas y poco consumo de frutas y verduras).

La importancia de los factores de riesgo tradicionales de las ECCV radica en que en su mayoría dependen de las conductas y formas de vida de las personas, conductas que al ser identificadas, deberían ser intervenidas, disminuyendo así su impacto y evitando la acumulación del riesgo que puede desencadenar en la generación de dichos eventos.

Los estudios han centrado sus esfuerzos en identificar dichos comportamientos en la población adulta, quienes por la edad y acumulación de riesgos tienen mayor probabilidad de presentar un episodio similar. En población joven y universitaria se referencian estudios de España y Chile (1999), Brasil (2011) y Argentina, para América Latina. En Colombia, los pocos estudios referenciados se circunscriben a ciudades como Cartagena (año 2009), Santa Fe de Bogotá y algunos poblacionales en Bucaramanga y Santiago de Cali (año 2005) que incluyen grupos de personas jóvenes.

Los hallazgos del presente estudio sobre factores de riesgo tradicionales modificables en jóvenes estudiantes de la facultad de salud son alarmantes. Prevalencias de eventos como la inactividad física y el consumo de licor son mayores que aquellos reportados en otros estudios. Se aplicaron pruebas de significancia para realizar comparaciones con estudios en Brasil (2011), Chile (1999) y Cartagena (2009) con las cuales se evidenció prevalencias superiores en inactividad física al comparar con estudios en Brasil, y más bajas para consumo de alcohol y cigarrillo al comparar con el estudio de Cartagena.
Aunque no se cuenta con información u otros estudios en población similar (enfermería - medicina) en factores de riesgo de ECCV, que pudieran ser comparados con los del presente estudio, se considera que los resultados son alarmantes. Se esperaría que la formación en salud y para la salud facilitara la adquisición de conocimientos y herramientas que les permitiera a los estudiantes, tomar decisiones sobre sus comportamientos de riesgo, ya que según Tobón (2011) "la educación y la información se relacionan con el desarrollo de habilidades personales para optar por decisiones saludables ${ }^{6 "}$.

Se supone entonces, que la educación y los conocimientos en el tema no son suficientes para la adquisición de hábitos saludables. El entorno desempeña un papel fundamental en los mismos y así como el hogar, el ambiente universitario es vital en la adquisición de prácticas saludables y en el desarrollo integral de los individuos.

Existen factores internos y externos a las personas que se relacionan con sus prácticas de autocuidado, según el Ministerio de la Protección Social (2014) "los conocimientos y la voluntad son factores internos; del mismo modo, los aspectos culturales, económicos, políticos y sociales son factores externos que determinan las prácticas de autocuidado ${ }^{7 \%}$.

Muchas universidades en Colombia han asumido su responsabilidad en el mejoramiento de las condiciones de salud y calidad de vida de sus integrantes y han conformado la red de universidades saludables. Si se tienen en cuenta que la población universitaria está compuesta en su mayoría por individuos jóvenes especialmente en su etapa de adolescencia y adultez temprana, época en la cual las personas afianzan sus conocimientos y prácticas y siguen como modelo a sus pares; la intención y compromiso de estas instituciones es la puesta en marcha de actividades en el marco de la escuela saludable y la responsabilidad so-

6. Tobón Correa, Ofelia. El autocuidado: una habilidad para vivir. Universidad de Caldas, programa de enfermería. Manizales, Caldas 2011.

7. Ministerio de la Protección Social. [Internet] [Consultado Mayo 2014]; Disponible en: URL: http://www.minproteccionsocial.gov.co 
cial que asume un centro de educación superior en la formación integral de personas, se presenta la oportunidad propicia para generar espacios de reflexión y trabajo en torno a las enfermedades cerebro-cardiovasculares y a sus factores de riesgo.

Se genera entonces, la necesidad de diseñar y adoptar planes y programas de intervención, educación e investigación en prevención de factores de riesgo de ECCV en las Universidades de la región y el país, dado que la Organización Panamericana de la Salud (2009) manifiesta que

"la única forma posible de abordar los distintos determinantes que deterioran la calidad de vida de la población es mediante la acción conjunta entre los diferentes actores sociales para transformar condiciones de vida de los miembros de la comunidad, potencializando sus capacidades individuales y colectivas en la producción de bienestar social ${ }^{8 "}$.

Se propone la generación de una política clara de universidad saludable contemplada en el proyecto educativo institucional y en cada uno de los proyectos educativos de sus facultades y programas, haciendo participes a todos los actores y dependencias universitarias, dado que

"una institución escolar no se convierte en escuela saludable por designación oficial de autoridades de salud o de educación, sino por decisión voluntaria de la comunidad educativa de promo- ver mejores condiciones de vida para todos sus miembros y por todos los medios a su alcance, según la OPS (2009) ${ }^{9 "}$

Un ambiente escolar que propicie espacios de participación para los estudiantes, que brinde conocimientos en estilos de vida saludable y que permita la promoción de habilidades y destrezas como herramientas de autocuidado, posibilita la toma de decisiones que conduzca a los jóvenes a generar hábitos saludables en pro del bienestar y la calidad de vida.

La vigilancia y el monitoreo en salud se convierten en una herramienta importante en el seguimiento de los eventos, la recolección sistemática, análisis e interpretación de información del mismo. Todos estos datos son indispensables en la planificación, implementación y evaluación de los programas de intervención.

Los resultados reflejados en el presente documento pueden ser útiles como línea de inicio, en la implementación de un programa de vigilancia y monitoreo de factores de riesgo de enfermedad cerebro-cardiovascular, dirigido a los estudiantes universitarios, que incluya estrategias de promoción y educación para la salud, prevención de la enfermedad e investigación, que permitan aumentar las capacidades y oportunidades de la comunidad, para mejorar la calidad de vida, el desarrollo integral y la construcción de entornos favorecedores de la salud, a través de la acción interdisciplinaria.
8. Organización Panamericana de la Salud OPS. Estrategia de escuelas saludables 2009. [Internet] [Consultado Marzo 2015]; Disponible en: http:// www.minsalud.gov.co/Documentos\%20y\%20Publicaciones/Entorno\%20 Saludable $\% 20 \mathrm{y} \% 20$ Desarrollo\%20Territorial.pdf
9. Organización Panamericana de la Salud OPS. Estrategia de escuelas saludables 2009. [Internet] [Consultado Marzo 2015]; Disponible en: http:// www.minsalud.gov.co/Documentos\%20y\%20Publicaciones/Entorno $\% 20$ Saludable\%20y\%20Desarrollo\%20Territorial.pdf 


\section{BIBLIOGRAFÍA}

1. American Heart Association. [Internet] 2012[Consultado marzo 2014]; Disponible en: URL: http://www.americanheart.org/presenter.jhtml?identifier $=3001008$

2. International Diabetes Federation. [Internet] [Consultado julio 2014] Disponible en: URL: http://www.idf.org

3. Texas Hearth Institute. [Internet] [Consultado 30 de Marzo 2015]Disponible en: URL: http:// www.texasheartinstitute.org/HIC/Topics_Esp/HSmart/riskspan.cfm

4. World Health Organization. Global status report on noncommunicable diseases 2010. WHO Library Cataloguing-in-Publication. 2011. ISBN 9789240686458

5. Tobón correa, Ofelia. El autocuidado: una habilidad para vivir. Universidad de Caldas, programa de enfermería. Manizales, Caldas 2011

6. Ministerio de la Protección Social. [Internet] [Consultado Mayo 2014] Disponible en: URL: http://www.minproteccionsocial.gov.co

7. Organización Panamericana de la Salud OPS. Estrategia de escuelas saludables 2009. [Internet] [Consultado Marzo 2015]; Disponible en: http://www.minsalud.gov.co/Documentos\%20 y\%20Publicaciones/Entorno\%20Saludable\%20y\%20Desarrollo\%20Territorial.pdf

8. Alcaldía de Santiago de Cali, Secretaría de Salud Pública Municipal, Universidad del Valle. Centro para el Desarrollo y Evaluación de Políticas y Tecnología en Salud Pública, CEDETES. Proyecto Diseño de un sistema de Vigilancia de Factores de Riesgo asociados a las Enfermedades Crónicas No Transmisibles ECNT. Estudio de Prevalencia de Factores de Riesgo Asociados a las ECNT Cali, 2005.

9. Beck, Carmen Cristina et al. Factores de riesgo cardiovascular en adolescentes en un municipio de Brasil: prevalencia y asociaciones con variables sociodemográficas. Revista Brasilera de epidemiología, Brasil, 2011; 14(1): 36-49.

10. Cazuza de Farías, José et al. Factores de riesgo cardiovascular en adolescentes: prevalencia en asociación con factores sociodemográficos. Revista Brasilera de epidemiología, Brasil, 2011, 14(1): 50-62.

11. Chiang Salgado, María Teresa. Casanueva Escobar, Víctor et al. Factores de riesgo cardiovascular en estudiantes universitarios chilenos. Revista de salud pública de México. México 1999, 41(6).

12. Guerrero C, Angélica. Machado J, Cindy. Factores de riesgo de enfermedades crónicas en estudiantes universitarios, Cartagena, Corporación Universitaria Rafael Núñez. Colombia 2009 
Copyright of Journal of Research of the University of Quindio is the property of Journal of Research of the University of Quindio and its content may not be copied or emailed to multiple sites or posted to a listserv without the copyright holder's express written permission. However, users may print, download, or email articles for individual use. 